\title{
Years of life lost as a measure of premature death among dual-chamber pacemaker recipients from Małopolska Province
}

\author{
Maciej Dębski', Irena Maniecka-Bryła², Elżbieta Dziankowska-Zaborszczyk², Mateusz Ulman'1, Andrzej Ząbek1,

 \\ 1 Department of Electrocardiology, John Paul II Hospital, Kraków, Poland \\ 2 Department of Epidemiology and Biostatistics, Division of Social and Preventive Medicine, Medical University of Lodz, Łódź, Poland \\ 3 Department of Anatomy, Jagiellonian University Medical College, Kraków, Poland \\ 4 Institute of Cardiology, Jagiellonian University Medical College, Kraków, Poland
}

\section{KEY WORDS}

average annual percentage change, pacemaker, premature mortality, years of life lost

\section{EDITORIAL}

page 659

\begin{abstract}
BACKGROUND Pacemakers have become the standard of care in patients with severe bradycardia and conduction abnormalities. The survival and premature mortality can be assessed using the years of life lost (YLLS).

AIMS The aim of the study was to analyze mortality trends over the period from 1999 to 2015 among patients implanted with a dual-chamber (DDD) pacemaker who were inhabitants of Małopolska Province. METHODS This was a retrospective study of records collected from consecutive patients who underwent de novo DDD pacemaker implantation at a single center between 1984 and 2014. Inclusion criteria were residence status in Małopolska Province at the latest follow-up visit and death between 1999 and 2015. The standard expected years of life lost per death was used to calculate YLLs. Time trends were evaluated with joinpoint models and presented as an average annual percentage change (AAPC).

RESULTS Among a total of 3932 consecutive patients implanted with a DDD pacemaker, 1211 patients met the inclusion criteria. We noted an increase in the mean age at implant from 70 years in 1999 to 75.5 years in 2015 (AAPC, $0.6 \% ; P<0.05$ ), the number of years lived after DDD pacemaker implantation from 2.6 years to 8.2 years (AAPC, $7.4 \% ; P<0.05$ ), and the mean age at death from 72.6 years to 83.8 years (AAPC, $0.89 \% ; P<0.05$ ). Finally, we observed a reduction of the YLLs per death from 17.4 years in 1999 to 9 years in 2015 (AAPC, $-4 \% ; P<0.05$ ). All trends were significant for both men and women.

CONCLUSIONS In the 17-year follow-up, we showed significant changes in analyzed trends, in particular a reduction in the YLLs per death.
\end{abstract}

INTRODUCTION As shown in the FOLLOW-PACE study by Udo et al, ${ }^{1}$ pacemakers implanted for slow heart rhythm restore life expectancy to normal levels. The results have suggested that the prognosis of a contemporary patient with a pacemaker was primarily determined by concomitant cardiovascular disease, and not by the rhythm disorder itself. Therefore, after pacemaker implantation for bradycardia, more attention should be paid to the detection and treatment of other concurrent cardiovascular conditions. Noteworthy, in a previous study, we showed that compared with the general population, the observed survival of dual-chamber (DDD) pacemaker recipients was significantly inferior until 12 years post implant, while after 12 years of follow-up, the observed survival was significantly higher. ${ }^{2}$ Death rates alone do not provide a complete picture of the burden that deaths impose on the population. Another useful measure, which has been gaining increasing popularity in epidemiological reports, 


\section{WHAT'S NEW?}

We investigated the lifespan of dual-chamber (DDD) pacemaker recipients living in Małopolska Province, Poland. We performed a trend analysis of age at implantation, survival after implantation, and age at death between 1999 and 2015. Furthermore, we evaluated premature mortality by calculating the years of life lost over a 17-year follow-up. The results demonstrated a continuous improvement in the survival after DDD pacemaker implantation and a significant reduction of the years of life lost. To our knowledge, this is the first report adopting an accurate and comprehensive measure that quantifies the level and trend of premature mortality in patients with permanent pacemaker.

is the years of life lost (YLLs). This less conventional indicator may add a different dimension to mortality trends in a population, in particular with regard to premature mortality. According to Eurostat, the YLL is the mortality component of the disability-adjusted life years, introduced by the World Bank and the World Health Organization as a description of the "burden of disease." The YLL ignores morbidity, and therefore only describes "the burden of mortality."3 The aim of the study was to use the YLL measure to analyze mortality trends in the period from 1999 to 2015 among inhabitants of Małopolska Province who were implanted with a DDD pacemaker.

METHODS This was a retrospective study of records collected from consecutive patients who underwent primary DDD pacemaker implantation at a high-volume tertiary reference implantation center between October 4, 1984, and December 31, 2014. Detailed methods of data collection and the baseline characteristics of the study population were presented previously. ${ }^{2,4}$ The inclusion criteria were residence status in Małopolska Province at the time of the last pacemaker follow-up visit and death between January 1, 1999, and December 31, 2015. Małopolska Province came into being on January 1, 1999. The data on the survival status of patients and the dates of death of deceased patients were collected from the national death registration system in 2016. The data used in the analysis included: 1) baseline demographic characteristics of patients: date of birth, age at implantation, and sex; 2) place of residence during follow-up; and 3) date of death declared in the death certificate. The following time trends were analyzed: 1) YLLs per 1 death; 2) mean age at the time of death;3) mean age at pacemaker implantation; and 4) mean time from implantation to death.

Statistical analysis Time trends of analyzed variables were evaluated using the Joinpoint Regression software developed by the Surveillance, Epidemiology and End Results program of the $\mathrm{Na}$ tional Cancer Institute (Joinpoint Regression Program, Version 4.1.1.1. October 2014; Statistical
Methodology and Applications Branch, Surveillance Research Program, National Cancer Institute, Bethesda, Maryland, United States, 2014). This method is an advanced version of linear regression, where the time trend is expressed with a broken line, which is a sequence of segments joined in Joinpoint. To confirm whether the changes were significant, the Monte Carlo permutation method was applied. We estimated the annual percentage change (APC) of the variables for each segment of the segmented regression curve, average annual percentage change (AAPC) as a summary measure of trend that applies over an entire time period, and their $95 \%$ confidence intervals (CIs). When the trend is constant over the entire period of interest, the AAPC will be equal to the estimated APC. ${ }^{5}$ In all analyses, a $P$ value of less than 0.05 was considered significant.

For YLL calculation, we used the standard expected years of life lost per death (SEYLLs) measure. It was used to calculate the number of YLLs by the studied population in comparison with the YLLs by the standard population. The SEYLL weighs mortality, age at death, and the population structure, counts all life years lost at all ages, as well as weighs death by the residual life expectancy in a standard life table. In the present study, the SEYLL index was calculated according to the Marshall method and expressed as a rate per a single death ${ }^{6}$ :

$$
\text { SEYLL }=\sum_{a=0}^{k} d_{a} e_{a}
$$

where $a$ denotes age at death; $k$, age of the oldest deceased patient; $d_{a}$ number of patients deceased at the age of $a$; and $e_{\mathrm{a}}$ number of expected years of life for a person at the age of $a$. The number of expected years of life $\left(e_{a}\right)$ for a person at the age of $a$ was calculated based on the standard life table presented by Murray et $\mathrm{al}^{7}$ in the Global Burden of Disease Study 2010.

RESULTS Out of 3932 patients, 3928 (99.9\%) had known survival status. At the time of the last follow-up visit, 3702 patients (94.2\%) were inhabitants of Małopolska Province. Among the inhabitants of this province, 1211 patients (32.7\%) died between 1999 and 2015, including 696 men (57.5\%) and 515 women (42.5\%) (TABlE 1). Out of 226 inhabitants of other provinces, 69 patients died. During follow-up, 7 of the 1211 patients had a device upgrade to an implantable cardioverter-defibrillator, 8 patients had an upgrade to cardiac resynchronization therapy pacemaker, and 3 patients had an upgrade to cardiac resynchronization therapy defibrillator. We observed an increase in the mean age at the time of implantation from 70 years in 1999 to 75.5 years in 2015 (AAPC, 0.62\%; 95\% CI, 0.4\%-0.9\%; $P<0.05$ ): in women, from 75.2 to 76.7 years (AAPC, $0.29 \%$; 


\begin{tabular}{lllllllllllllllllll} 
Sex & \multicolumn{110}{c}{ Year } \\
& 1999 & 2000 & 2001 & 2002 & 2003 & 2004 & 2005 & 2006 & 2007 & 2008 & 2009 & 2010 & 2011 & 2012 & 2013 & 2014 & 2015 & Total \\
Women & 6 & 6 & 13 & 11 & 7 & 13 & 19 & 22 & 21 & 31 & 29 & 32 & 53 & 65 & 54 & 60 & 73 & 515 \\
\hline Men & 22 & 19 & 17 & 22 & 19 & 23 & 27 & 28 & 43 & 50 & 55 & 49 & 55 & 57 & 72 & 73 & 65 & 696 \\
\hline Total & 28 & 25 & 30 & 33 & 26 & 36 & 46 & 50 & 64 & 81 & 84 & 81 & 108 & 122 & 126 & 133 & 138 & 1211 \\
\hline
\end{tabular}

95\% CI, 0.1\%-0.5\%; $P<0.05)$; in men, from 68.6 to 74.2 years (AAPC, $0.72 \%$; $95 \% \mathrm{CI}, 0.4 \%-1 \%$; $P<0.05)$ (FIGURE 1 ).

In the period from 1999 to 2015, we observed a prolonged life span after primary DDD pacemaker implantation, both for women and men. In 1999, the survival after pacemaker implantation was 2.6 years ( 2.4 years in women and 2.7 years in men), whereas in 2015, it reached 8.2 years (7.3 years in women and 9.3 years in men). The average rise in the number of survived years after

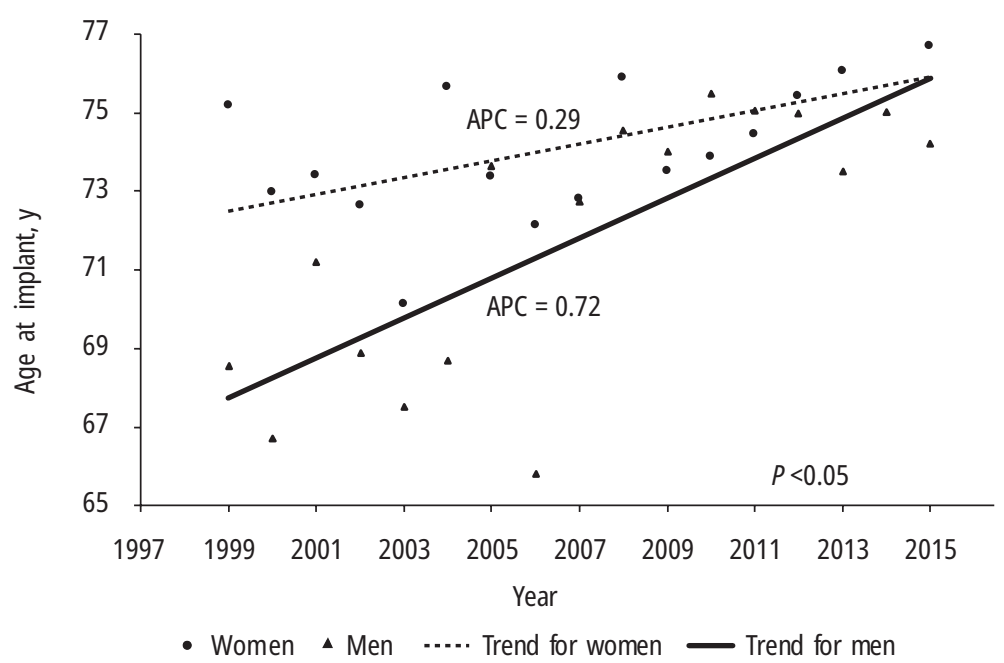

FIGURE 1 Age at the time of primary DDD pacemaker implantation Abbreviations: APC, annual percentage change

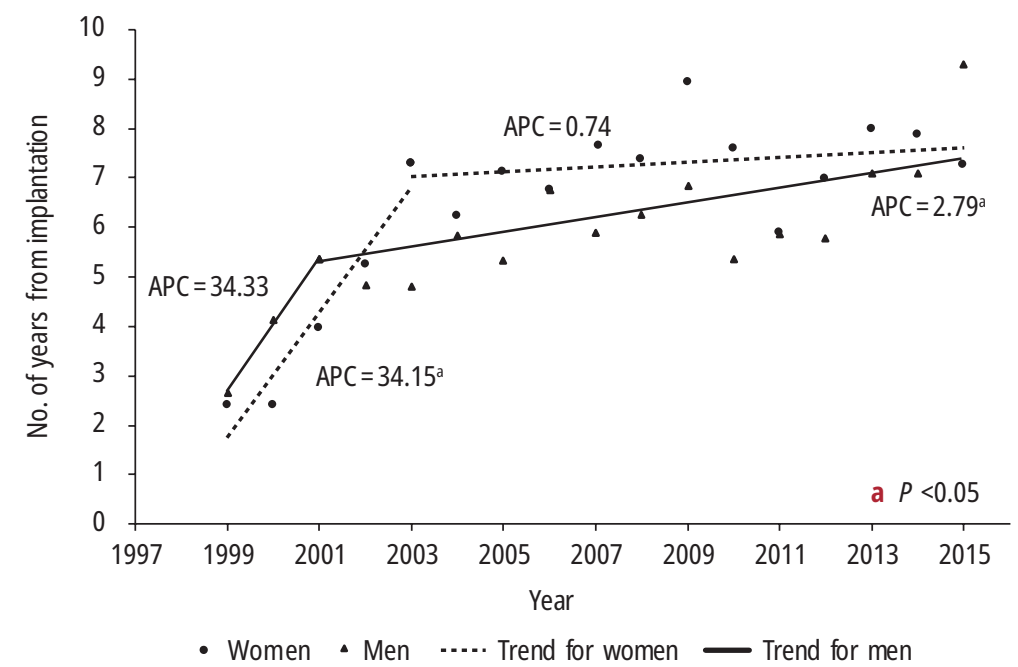

FIGURE 2 Average lifespan following DDD pacemaker implantation Abbreviations: see FIGURE 1 pacemaker insertion calculated as AAPC was 7.4\% (95\% CI, 4.2\%-10.7\%; $P<0.05)$ in the whole study group, $8.2 \%$ (95\% CI, 5\%-11.5\%; $P<0.05)$ in women, and $6.3 \%$ (95\% CI, $1.7 \%-11.1 \% ; P<0.05)$ in men. Among women who died between 1999 and 2003 and men who died between 1999 and 2001 , the APC of postimplant survival reached $34.2 \%$ (95\% CI, $19.4 \%-50.8 \% ; P<0.05)$ and $34.3 \%$ (95\% CI, $-7.7 \%$ to $95.5 \%$; $P>0.05$ ), respectively. For women who died between 2004 and 2015 and for men who died between 2002 and 2015, the APC was lower and amounted to $0.7 \%(95 \% \mathrm{CI}$, $-1.4 \%$ to $3 \%$; $P>0.05)$ and $2.8 \%$ (95\% CI, $1 \%-4.6 \%$; $P<0.05$ ), respectively (FIGURE 2 ).

The mean age at the time of death increased at a rate of $0.9 \%(95 \% \mathrm{CI}, 0.7 \%-1 \% ; P<0.05)$; $0.6 \%$ (95\% CI, $0.4 \%-0.8 \%$; $P<0.05$ ) for women and $1 \%$ (95\% CI, $0.6 \%-1 \%$; $P<0.05)$ for men. The mean age of deceased pacemaker recipients in 1999 was 72.6 years (77.6 years in women and 71.2 years in men), whereas in 2015 , it was 83.8 years (84 years in women and 83.5 years in men) (FIGURE3). Finally, we observed a decrease in YLLs per death with an AAPC of $-4 \%$ (95\% CI, $-4.9 \%$ to $-3.1 \%$; $P<0.05)$ : for women, $-2.9 \%$ (95\% CI, $-3.9 \%$ to $-2 \%$; $P<0.05$ ), and for men, $-4.2 \%$ (95\% CI, $-5.2 \%$ to $-3.1 \%$; $P<0.05)$. The YLLs per death in 1999 amounted to 17.4 years (13.3 in women and 18.6 in men), whereas in 2015, the value dropped to 9 (8.8 in women and 9.2 in men) (FIGURE 4).

DISCUSSION In this study, we demonstrated trends over the 17-year period (1999-2015) in the characteristics and outcomes of DDD pacemaker recipients living in Małopolska Province in Poland. We observed shifts toward DDD pacemaker implantation at an older age, extended longevity after primary implant, and reduction in the YLLs per death. Furthermore, we noted that the APC of YLL reduction was greater in men than in women. Our findings provide robust evidence for trends in demographics and survival among a large group of DDD pacemaker recipients.

Few studies have investigated the epidemiology of patients with dual-chamber pacing. Our previous findings demonstrated that women were older than men at the time of pacemaker implantation and that an average age at implant increased over the 3 decades. Similar trends 


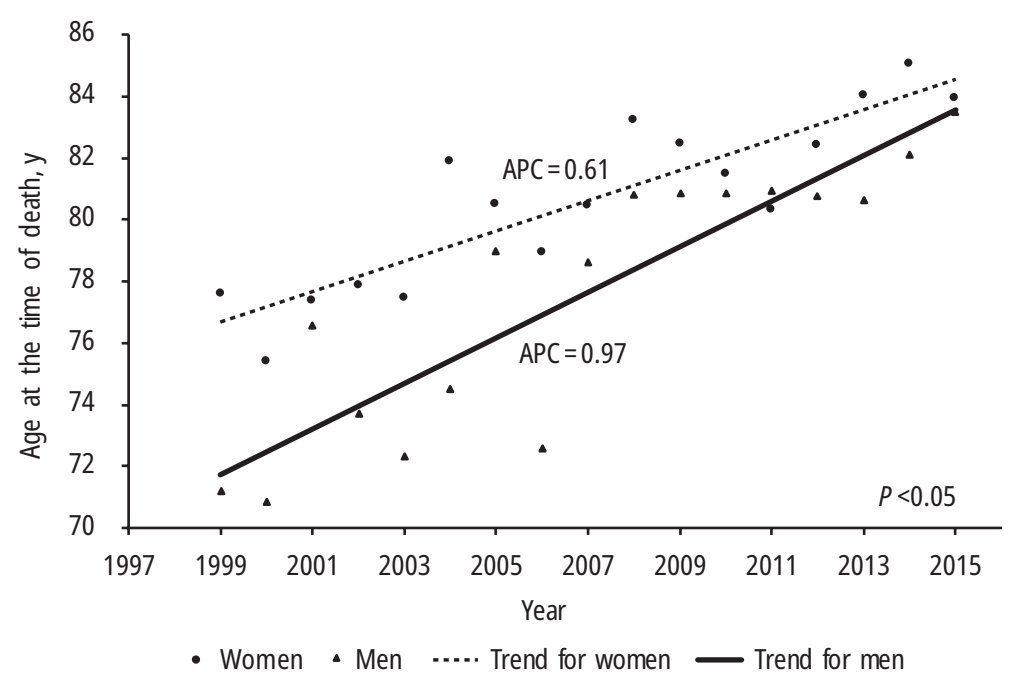

FIGURE 3 Average age at the time of death

Abbreviations: see FIGURE 1

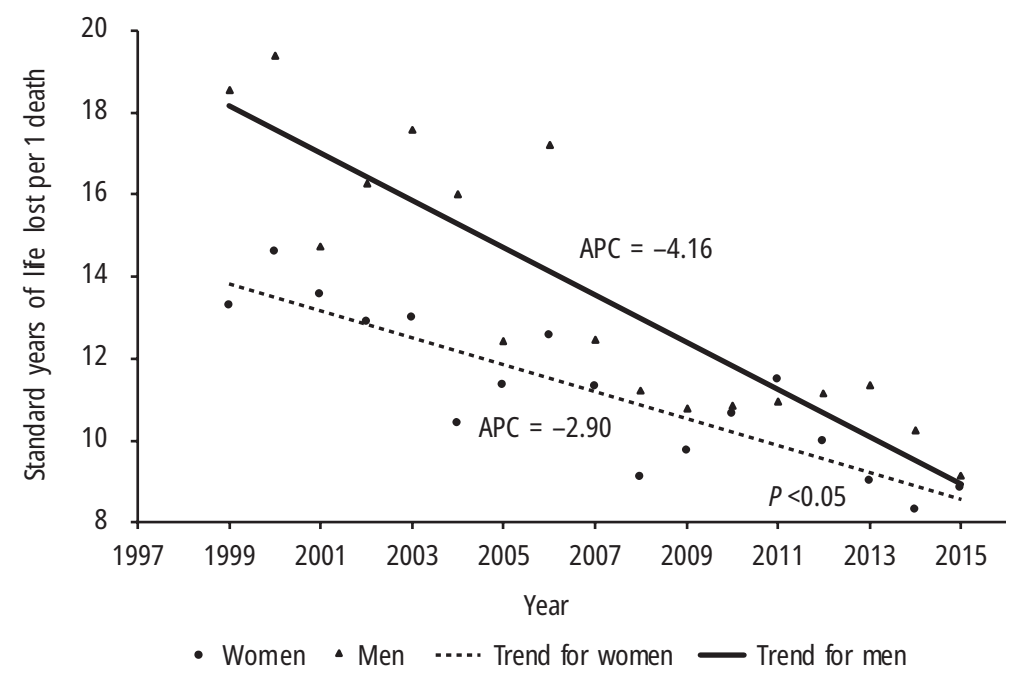

FIGURE 4 Years of life lost per death

Abbreviations: see FIGURE 1 changes reflecting Poland's progress between 1999 and 2015.

The study by the Global Burden of Disease 2017 sheds some light on global and regional changes in mortality between 1980 and 2017. ${ }^{16}$ In general, YLL rates decreased while a sociodemographic index for a given region increased. The total number of deaths due to noncommunicable diseases, including both cardiovascular diseases and neoplasms, has risen steadily since 1990, driven by aging and population growth, while death and YLL rates have been falling consistently as a result of improvements in prevention strategies and healthcare. In Poland, the observed life expectancy between 2000 and 2017 rose from 78 to 81.8 years for women and from 69.6 to 74.1 years for men. ${ }^{17}$ Noncommunicable diseases contributed to $76.9 \%$ of YLLs among men and $87.5 \%$ of YLLs among women in $2000 . .^{18}$ The total all-cause mortality rate for the age group of 70 years or older in 1999 was 7273 deaths per 100000 , and in 2015, it dropped to 6361 deaths per 100000 . The rate of deaths attributable to cardiovascular diseases in this population decreased from 4226 deaths per 100000 in 1999 to 3215 deaths per 100000 in 2015. Between 2000 and 2014, the rates of the YLLs per death due to cardiovascular diseases dropped, with an AAPC of $-0.5 \%$ among men and $-1.4 \%$ among women. ${ }^{19}$ In the present study, the pace of decline in YLLs per death was significantly higher, with an AAPC of $-4.2 \%$ for men and of $-2.9 \%$ for women across the 17-year follow-up. This remarkable difference may be explained by close and mostly life-long follow-up of pacemaker recipients by highly specialized cardiologists in a tertiary reference center that provides comprehensive cardiac and cardiothoracic care and access to other medical and surgical facilities.

Interestingly, the observed significant rise in the lifespan after implant in women between 1999 and 2003, with an APC over 34\%, corresponded to a significant drop in the SEYLL per living person due to chronic noncommunicable diseases among the inhabitants of Poland in the period from 2000 to $2003 .{ }^{18}$ The trend for men in the period from 1999 to 2001 was not significant, most likely due to the small sample. Since 1990, the 2 leading causes of death in Poland have continued to be ischemic heart disease and stroke. Noteworthy, the rate of YLLs per death secondary to ischemic heart disease decreased significantly in the years 2000 to 2014 , with an AAPC of $-1 \%$ for men and $-1.2 \%$ for women, while the rate of YLLs per death secondary to cerebrovascular diseases decreased at a pace of $-0.5 \%$ for men and $-1.3 \%$ for women. ${ }^{19}$ On the other hand, the number of YLLs has increased for lung cancer, colorectal cancer, and Alzheimer disease. Importantly, according to the Global Burden of Disease, the Healthcare Access and Quality Index in Poland had 
significantly improved between 2000 and 2016, with an AAPC of $0.9 \%$. This measure is based on risk-standardized mortality rates or mortality-to-incidence ratios from causes that, in the presence of quality healthcare, should not result in death (also known as amenable mortality). Additionally, the changes in behavioral, metabolic, and occupational risk that occurred in Poland between 2007 and 2017 have resulted in lower rates of deaths and disability due to tobacco smoking, dietary risks, hypertension, and hypercholesterolemia, whereas high body mass index and high fasting plasma glucose levels have contributed to more deaths and disability.

Our findings should be interpreted in the context of limitations imposed by a retrospective study design. Due to the lack of data on the cause of death, we were unable to perform comparisons with the general population. Moreover, the results cannot be generalized to a general pacemaker population as our sample consisted only of DDD pacemaker recipients. In addition, we included only patients who were deceased and were inhabitants of Małopolska Province at the last follow-up visit and excluded patients who remained alive until 2015 or were inhabitants of other provinces. On the other hand, the life expectancy may vary across regions in Poland; therefore, by narrowing the sample down, we aimed to obtain the most accurate estimation of patients' demographic characteristics and lifespan. Nonetheless, we believe that the ability to generate locally representative estimates of demographic and survival trends in DDD pacemaker population is the strength of our study.

In conclusion, we identified a shift toward favorable life expectancy and a decrease in the YLLs among DDD recipients in Małopolska Province. Many of the factors driving that positive trend include state-of-the-art cardiology care during follow-up but also economic transformation, improvement in general healthcare system, as well as behavioral and lifestyle changes observed in Poland.

\section{ARTICLE INFORMATION}

\section{CONFLICT OF INTEREST None declared.}

OPEN ACCESS This is an Open Access article distributed under the terms of the Creative Commons Attribution-NonCommercial-NoDerivatives 4.0 International License (CC BY-NC-ND 4.0), allowing third parties to download articles and share them with others, provided the original work is properly cited, not changed in any way, distributed under the same license, and used for noncommercial purposes only. For commercial use, please contact the journal office at kardiologiapolska@ptkardio.pl.

HOW TO CITE Dębski M, Maniecka-Bryła I, Dziankowska-Zaborszczyk E, et al. Years of life lost as a measure of premature death among dual-chamber pacemaker recipients from Małopolska Province. Kardiol Pol. 2019; 77: 683-687. doi:10.33963/KP.14839

\section{REFERENCES}

1 Udo EO, van Hemel NM, Zuithoff NP, et al. Prognosis of the bradycardia pacemaker recipient assessed at first implantation: a nationwide cohort study. Heart. 2013; 99: 1573-1578.

2 Dębski M, Ulman M, Ząbek A, et al. Association of selected factors with long-term prognosis and mortality after dual-chamber pacemaker implant. Cardiol J. 2018. [Epub ahead of print].
3 Database. Eurostat website. http://ec.europa.eu/eurostat/data/database. Accessed December 2018.

4 Dębski M, Ulman M, Ząbek A, et al. Lead-related complications after DDD pacemaker implantation. Kardiol Pol. 2018; 76: 1224-1231.

5 Clegg LX, Hankey BF, Tiwari R, et al. Estimating average annual per cent change in trend analysis. Stat Med. 2009; 20: 3670-3682.

6 Marshall RJ. Standard expected years of life lost as a measure of disease burden: an investigation of its presentation, meaning and interpretation. In: Preedy VR, Watson RR, eds. Handbook of disease burdens and quality of life measures. Springer; 2009: 3421-3434.

7 Murray Ch J L, Ezzati M, Flaxman AD, et al. GBD 2010: design, definitions, and metrics. Lancet. 2012; 380: 2063-2066.

8 Bradshaw PJ, Stobie P, Knuiman MW, et al. Life expectancy after implantation of a first cardiac permanent pacemaker (1995-2008): a population-based study. Int J Cardiol. 2015; 190: 42-46.

9 Brunner M, Olschewski M, Geibel A, et al. Long-term survival after pacemaker implantation. Prognostic importance of gender and baseline patient characteristics. Eur Heart J. 2004; 25: 88-95.

10 Greenspon AJ, Patel JD, Lau E, et al. Trends in permanent pacemaker implantation in the United States from 1993 to 2009: increasing complexity of patients and procedures. J Am Coll Cardiol. 2012; 60: 1540-1545.

11 Mond HG, Proclemer A. The 11th world survey of cardiac pacing and implantable cardioverter-defibrillators: calendar year 2009-a World Society of Arrhythmia's project. Pacing Clin Electrophysiol. 2011; 34: 1013-1027.

12 Nowak B, Misselwitz B, Erdogan A, et al. Do gender differences exist in pacemaker implantation? Results of an obligatory external quality control program. Europace. 2010; 12: 210-215.

13 Uslan DZ, Tleyjeh IM, Baddour LM, et al. Temporal trends in permanent pacemaker implantation: a population-based study. Am Heart J. 2008; 155: 896-903.

14 Jelić V, Belkić K, Djordjević M, Kocović D. Survival in 1,431 pacemaker patients: prognostic factors and comparison with the general population. Pacing Clin Electrophysiol. 1992; 15: 141-147.

15 Mayosi BM, Little F, Millar RN. Long-term survival after permanent pacemaker implantation in young adults: 30 year experience. Pacing Clin Electrophysiol. 1999; 22: 407-412.

16 GBD 2017 Causes of Death Collaborators. Global, regional, and national age-sex-specific mortality for 282 causes of death in 195 countries and territories, 1980-2017: a systematic analysis for the Global Burden of Disease Study 2017. Lancet. 2018; 392: 1736-1788.

17 Poland. IHME website. http://www.healthdata.org/poland. Accessed December 2018.

18 Pikala M, Maniecka-Bryła I. Fifteen-year mortality trends in Poland analysed with the use of standard expected years of life lost, 2000-2014. Sci Rep. 2017; 18: 8730 .

19 Pikala M, Maniecka-Bryła I. Fifteen-year mortality trends due to cardiovascular diseases in Poland using standard expected years of life lost, 2000-2014. Kardiol Pol. 2017; 75: 1033-1040. 